\title{
Location, location, location: it's all in the timing for replication origins
}

\author{
Oscar M. Aparicio ${ }^{1}$ \\ Molecular and Computational Biology Program, University of Southern California, Los Angeles, California 90089, USA
}

The differential replication timing of eukaryotic replication origins has long been linked with epigenetic regulation of gene expression and more recently with genome stability and mutation rates; however, the mechanism has remained obscure. Recent studies have shed new light by identifying novel factors that determine origin timing in yeasts and mammalian cells and implicate the spatial organization of origins within nuclear territories in the mechanism. These new insights, along with recent findings that several initiation factors are limiting relative to licensed origins, support and shape an emerging model for replication timing control. The mechanisms that control the spatial organization of replication origins have potential impacts for genome regulation beyond replication.

\section{Scope \\ Several excellent reviews have recently examined the significance of replication timing in epigenetic gene regulation and reprogramming, genetic instability, and mutation rates and evolution (for review, see Aladjem 2007; Gondor and Ohlsson 2009; Mendez 2009; Gilbert et al. 2010; Natsume and Tanaka 2010; Burgess 2011; Herrick 2011). Despite substantial progress in character- izing replication timing in various model systems, our understanding of the underlying mechanisms that estab- lish the timing of origin activation has remained rather vague. This review highlights recently published studies that provide new mechanistic insights by identifying novel factors that control replication timing throughout the budding yeast and fission yeast genomes. These factors are conserved in higher eukaryotes, and one has already been shown to control replication timing in mammalian genomes, where replication timing is linked with gene regulation and the developmental program.}

\section{Replication origins and initiation control}

The discovery of autonomously replicating sequences (ARSs) in the budding yeast Saccharomyces cerevisiae

[Keywords: replication timing; chromatin; Fkh1; Fkh2; Rif1; Taz1] ${ }^{1}$ Correspondence

E-mail oaparici@usc.edu

Article is online at http://www.genesdev.org/cgi/doi/10.1101/gad.209999.112. enabled the first detailed studies of chromosomal DNA replication origins in eukaryotic cells (Stinchcomb et al. 1979). ARSs were identified as relatively short (100- to 200-base-pair [bp]) chromosomal DNA sequences that confer replication to plasmids, allowing their mitotic propagation (for review, see Newlon and Theis 1993). Further analysis of these sequences within their native chromosomal loci confirmed their function as replication origins within chromosomes. As prototypes for replication origins in higher cells, ARS studies in yeast propelled much of the genetic and biochemical elucidation of the macromolecular events involved in replication initiation in all eukaryotes. Although yeast origins differ in sequence specificity from mammalian origins, both share highly conserved regulation at the molecular and cellular levels (for review, see Cvetic and Walter 2005).

Eukaryotic replication origins are governed by a series of protein recruitments, beginning with binding of the origin recognition complex (ORC), which marks potential sites of origin assembly (for review, see Bell and Dutta 2002). In G1 phase, ORC, Cdc6, and Cdt1 together load inactive minichromosome maintenance (MCM) helicase complexes at origins. This MCM loading step, which is also referred to as "licensing" or "pre-RC assembly," potentiates origins for initiation. Initiation is controlled by Dbf4-dependent kinase (DDK) and cyclin-dependent kinase (CDK), which are sequentially activated during the G1-to-S-phase transition (for review, see Labib 2010). DDK acts first, phosphorylating MCM subunits to load Cdc45 and Sld3 (Heller et al. 2011). Next, CDK phosphorylates Sld3 and Sld2, leading to the recruitment of GINS (which, together with Cdc45, stimulates MCM helicase) and additional factors, including DNA polymerases, to assemble a complete replisome and initiate DNA synthesis. Whereas this requisite sequence of events is shared by all active origins, they differ in timing and efficiency of initiation during $S$ phase.

Timing and efficiency are distinct measures that are frequently used to characterize origin activity. These measures are population averages and are typically determined through distinct methods (Kalejta and Hamlin 1996; Huberman 1997; van Brabant et al. 1998). Because origins differ in their characteristic initiation timing and exhibit substantial variance in this timing across a population, replication forks from adjacent origins sometimes replicate origins "passively," precluding initiation. 
Thus, most replication timing measurements represent a composite of initiation time and passive replication time. Efficiency is the resulting frequency of initiation (Friedman and Brewer 1995; Kalejta and Hamlin 1996). The replication timing and efficiency of neighboring origins are interrelated in that earlier or more efficient origins will often passively replicate their later or less efficient neighbors. Consequently, timing and efficiency are correlated with earlier origins generally being more efficient and later origins being less efficient (Yamashita et al. 1997; Yang et al. 2010). Both timing and efficiency may be thought of as reflecting the probability of origin firing, with early timing reflecting a high probability per unit time (at least in early S phase) and high efficiency reflecting a high, cumulative probability over the entire period of S phase. It has been suggested, therefore, that timing and efficiency derive from a common underlying mechanism that determines these probabilities (Rhind 2006). The central challenge has been to define this mechanism.

\section{Chromosomal position and chromatin structure}

Chromosomal context and local chromatin structure are major determinants of replication origin timing and efficiency, as demonstrated by seminal ARS relocation experiments (Ferguson and Fangman 1992). This study found that ARS501 residing near a subtelomeric region initiated replication later than $A R S 1$ residing near a centromere (CEN). Both origins initiated efficiently, indicating that both were efficiently licensed and competent to fire. Relocating $A R S 1$ to a subtelomeric location near ARS501 delayed its replication, whereas ARS501 initiated early when relocated outside of the subtelomeric region (onto a plasmid). These findings suggested that early firing is the normal state and that late timing is imposed by repressive chromatin flanking the origin, such as subtelomeric heterochromatin. Supporting and extending this idea, late-firing origins were identified in nontelomeric regions that fired early when located on a plasmid but fired later when $6-15 \mathrm{~kb}$ of native, flanking DNA was included on the plasmid, with the length of delay roughly proportional to the amount of flanking DNA (Friedman et al. 1996). Thus, chromosomal environment influences origin timing.

In some chromosomal contexts, origin firing is suppressed to the point of origin dormancy (Dubey et al. 1991). This phenomenon suggested the possibility that dormancy might result from interference with origin licensing. However, analysis of ARS301-a dormant origin associated with the heterochromatic, silent mating type locus $H M L$-indicated that $A R S 301$ was licensed and competent to initiate replication (Santocanale et al. 1999; Vujcic et al. 1999). Indeed, ARS301 initiated replication when given additional time, which was achieved by blocking its passive replication with hydroxyurea or deletion of flanking origins. Under these circumstances, $A R S 301$ fired very late, suggesting that a robust delay of firing is sufficient to cause dormancy by favoring passive replication.
Due to differences in origin efficiencies and timings, different subsets of origins are used in different cells of a population (Fangman and Brewer 1991; Kalejta and Hamlin 1996; Patel et al. 2006; Czajkowsky et al. 2008). Nevertheless, at the population level, replication timing profiles emerge reflecting individual origins' timings and efficiencies. Complete analysis of origin timings and efficiencies of all known origins on S. cerevisiae chromosomes III and VI, confirmed by whole-genome studies, has revealed general features of chromosomal replication, including early replication of at least one origin near each CEN, late firing and/or dormancy of origins in subtelomeric regions as well as those associated with the heterochromatic silencers, and a wide range of origin timings and efficiencies throughout other chromosomal regions, with some origins notably earlier than the CEN-proximal origins (Newlon et al. 1993; Friedman et al. 1997; Yamashita et al. 1997; Poloumienko et al. 2001; Raghuraman et al. 2001; Yabuki et al. 2002). Together with the ARS relocation studies, these findings implicated some aspect of these different chromosomal domains, such as chromatin structure, transcription, or subnuclear localization, in origin control.

The idea that chromatin structure, subnuclear localization, and/or gene expression regulate initiation gained direct support from studies that analyzed replication in strains with deletions of chromatin modifiers and related factors, such as SIR3, which is required for assembly of subtelomeric heterochromatin; YKU70, which is required for telomere localization to the nuclear periphery; and $R P D 3$, which deacetylates histones to regulate gene expression. Deletion of SIR3 resulted in earlier replication of subtelomeric origins, and when early origin $A R S 1$ was transplanted into a subtelomeric region, its timing was delayed in a SIR3-dependent manner (Stevenson and Gottschling 1999). Deletion of YKU70 also advanced replication timing of subtelomeric origins, suggesting a role for localization of subtelomeric chromatin to the nuclear periphery (Cosgrove et al. 2002). Moreover, deletion of RPD3 advanced the timing of most nontelomeric, late-firing origins, and tethering GCN5 histone acetylase to a late-firing origin advanced its initiation timing, indicating that histone acetylation levels surrounding origins influence origin activity (Vogelauer et al. 2002; Aparicio et al. 2004; Knott et al. 2009). However, increased histone acetylation by GCN5 targeting or $R P D 3$ deletion did not cause late origins to fire as early as the earliest-firing origins in the genome, suggesting the existence of unidentified, early-firing determinants. Recent studies in yeast have revealed such determinants.

\section{CENs impart early timing to proximal origins}

The finding that yeast CENs replicate early suggested that CENs might themselves influence replication timing; however, it was also possible that CENs reside in early-replicating regions but do not determine the timing of the regional origins. Studies of CEN replication timing in the related budding yeast Candida albicans provided 
further evidence that the presence of a functional CEN establishes early replication timing (Koren et al. 2010). In C. albicans, ablation of the CEN sequence on a chromosome resulted in the epigenetic formation of a functional, new CEN (neo-CEN) at a different site on the chromosome. Remarkably, a new origin was formed near the neoCEN through novel recruitment of ORC to the site, and the origin fired early. This occurred at several different neo-CEN loci in different strains, consistent with the neo-CEN establishing early timing; however, the possibility remained that these sites preferentially formed neoCENs due to the presence of early determinants and/or cryptic origins.

Recent experiments analyzing the effect of relocating a CEN within a chromosome recently provided direct evidence that CENs promote the earlier activation of proximal origins in S. cerevisiae (Pohl et al. 2012). This study analyzed a strain with a chromosomal rearrangement that relocated the CEN from its normal, early-firing locus next to a late-firing origin. This rearrangement advanced the late origin's timing while delaying activation of an origin near the original CEN locus. The advanced timing was dependent on the insertion of a functional CEN, demonstrating that the effect was not caused by extraneous sequences associated with the transplanted CEN. The effect of the CEN on replication timing was limited in cis to $\sim 19 \mathrm{~kb}$, and hence CENs do not account for the timing of most early origins genome-wide.

CENs also replicate early in the fission yeast Schizosaccharomyces pombe, although the mechanism appears to be quite distinct from that of budding yeast, which remains undefined. S. pombe CENs are substantially different in chromatin structure from S. cerevisiae CENs, undergoing H3K9 methylation, to which Swi6 (equivalent to metazoan HP1, which is apparently absent in $S$. cerevisiae) is recruited (Bannister et al. 2001; Nakayama et al. 2001). Similar heterochromatin exists in subtelomeric regions, which is at least partly responsible for late replication of subtelomeric domains. However, at pericentric origins and at the early-replicating, heterochromatic matK mating type locus, Swi6 directly binds and recruits Dfp1, the $S$. pombe equivalent of the DDKactivating subunit Dbf4 (Hayashi et al. 2009). Tethered recruitment of Dfp1 to the pericentric region can bypass the requirement of Swi6 for early firing of the pericentric origins. Thus, Swi6-mediated recruitment of DDK specifically ensures the early replication of CENs and matK in fission yeast, despite their heterochromatic structure, which otherwise would delay their replication. Swi6 was not responsible for early firing of a euchromatic origin.

\section{Global determinants}

Forkhead box (Fox) transcription factors Fkh1 and Fkh2 (Fkh1/2) have now been shown to account for the timing of most early (and late) origins in the budding yeast genome (Knott et al. 2012). Fkhl/2-binding sites were discovered as an anti-correlated genomic feature with the global set of Rpd3-regulated (late) origins (Knott et al. 2009), suggesting that Fkh1/2 might regulate replication timing. Indeed, cells lacking $F K H 1$ and $F K H 2$ exhibited profound changes in chromosomal replication profiles with significantly delayed firing of 106 normally early origins (termed Fkh-activated) and significantly advanced firing of 82 normally late origins (Fkh-repressed) out of 352 total origins detected. Strikingly, CEN-proximal origins (within $25 \mathrm{~kb}$ ) did not depend on Fkh1/2 for their early replication, consistent with the conclusion that CENs intrinsically determine origin timing locally. Fkh1/2 consensus binding sites were found to be enriched near Fkh-activated origins, and deletion of a pair of Fkh1/2binding sites proximal to one of these (ARS305) specifically delayed its firing in otherwise wild-type cells, demonstrating that Fkh1/2 act directly in cis to advance origin timing (Fig. 1A). In contrast, Fkh1/2 consensus binding sites were found to be depleted near Fkhrepressed origins, suggesting that the earlier firing of these origins derives indirectly from the delayed firing of

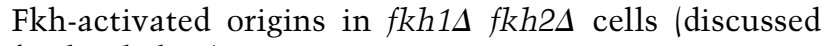
further below).

Two recent studies have identified telomere-binding proteins Rif1 and Taz1 as regulators of replication timing in the fission yeast S. pombe (Hayano et al. 2012; Tazumi et al. 2012). Tazl binds telomeric repeat sequences directly and binds Rif1, thus recruiting Rif1 to telomeres (Kanoh and Ishikawa 2001). Although telomeric chromatin has been implicated previously in replication timing control of subtelomeric regions, Rif1 and Taz1 are now found to regulate replication timing of numerous internal chromosomal loci as well. Tazumi et al. (2012) discovered a replication timing control (RTC) sequence adjacent to an internal, late-firing origin (AT2088/ars745) and showed that deletion of this site advanced origin timing (Fig. 1B). Furthermore, introduction of two copies of the 25-bp RTC sequence next to an early origin (ars2004) delayed its firing. The RTC contains a Taz1-binding sequence (a tandem repeat of telomere sequence), and deletion of $\operatorname{taz}^{+}$advanced the firing time of 26 (of 110) internal and 46 (of 46) subtelomeric late or dormant origins. RTCs were identified near 13 of the 26 internal origins, and chromatin immunoprecipitation (ChIP) analysis showed Tazl binding to all 13 of the internal origins with an RTC but not to 10 of the 13 deregulated origins lacking an apparent RTC. Similarly, Tazl binding was most enriched near the telomeres and did not appear to bind some of the more internal, subtelomeric origins that were deregulated by its deletion. Taz1 was suggested to act from a distance to regulate these origins.

Rif1 plays an even broader role in replication timing control. Hayano et al. (2012) discovered that deletion of rif $1^{+}$suppressed the lethality of a deletion of $h s k 1^{+}$, the catalytic subunit of DDK, suggesting that Rif1 acts in opposition to DDK in origin firing. Deletion of rif1 $1^{+}$ resulted in delayed activation of 134 normally early-firing origins (designated "EL" for early to late) and advanced activation timing of 189 normally late-firing origins ("LE" for late to early), out of 1083 origins detected; the LE group included all of the Tazl-regulated origins plus an additional 47 internal late or dormant origins. ChIP-chip analysis showed Rif1 binding near telomeres, as expected, 
Aparicio

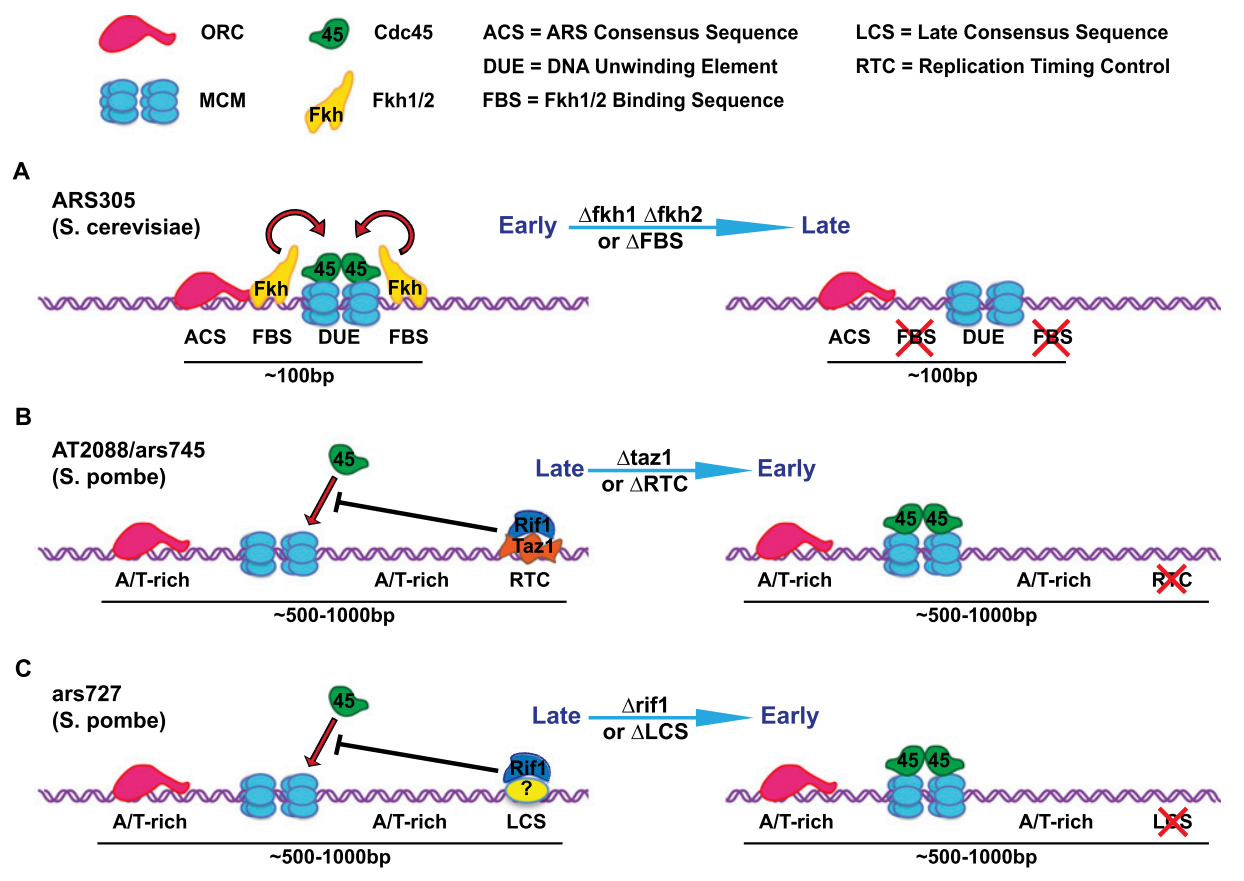

Figure 1. Yeast replication origins and factors regulating initiation timing. Structure models of origins regulated by Fkh1/2 $(A)$, Taz1Rif1 $(B)$, or Rif1 $(C)$. The left panels show regulation of Cdc45 recruitment in wild-type cells in G1/early S phase and resulting initiation timing, and the right panels show the effects of deleting indicated genes or protein-binding sites on Cdc45 recruitment and initiation timing. Arrows and blocking arrows regulating Cdc45 recruitment are not implied to act directly. DNA sequence elements and DNAbinding proteins are defined in the key. Rif1 is hypothesized to bind LCS directly or indirectly.

but also at many internal loci, where Rif1 binding was independent of Taz1. Rif1-binding sites mapped most frequently and closely to LE origins and least frequently and closely to EL origins, suggesting that Rif1 acts directly at LE origins to delay their activation and that the effect on EL origins occurs over a distance or is indirect (discussed further below). A significant exception, however, occurred at pericentric origins, which are in the EL group and bind Rif1 robustly, suggesting a direct effect on the timing of these origins. Whether Rif1 interacts with Swi6 to promote early firing of pericentric origins is unclear. Although it is not yet understood how Rif1 binds the Tazl-independent internal sites, Hayano et al. (2012) found a sequence motif associated with about half of the Rifl-binding sites near LE origins. This sequence is very similar to the previously identified late consensus sequence (LCS) responsible for late firing of ars727 (Yompakdee and Huberman 2004), a Rif1-bound LE origin (Fig. 1C); however, it remains to be tested whether the LCS is required for Rif1 binding, directly or indirectly. Despite this uncertainty, the data suggest that Rif1 acts directly to delay the firing of many internal and subtelomeric origins while also promoting the early firing of pericentric origins.

The role of Rif1 in replication timing control appears to be broadly conserved. Although Tazl is not present in S. cerevisiae, its function in recruiting Rif1 to telomeres is carried out by Rap1, which binds telomeres directly (Marcand et al. 1997). Budding yeast cells lacking Rif1 exhibit advanced replication timing of subtelomeric origins (Lian et al. 2011). This effect of RIF1 deletion was attributed to loss of the Rif1-dependent telomere lengthsensing mechanism, in which critically short telomeres are replicated early in contrast to the late replication of normal-length telomeres (for review, see Shore and Bianchi 2009). Similarly, the effect of YKU7O deletion on replication timing was attributed to telomere shortening in yku704 cells causing activation of the telomere lengthsensing mechanism. However, the finding that Rif1 also regulates timing of internal origins in fission yeast suggests a simpler mechanism in which shorter telomeres result in reduced Taz1 and Rif 1 binding at telomeres, which is directly required for late replication timing of subtelomeric origins. The budding yeast Rif1 study analyzed only a handful of origins, so the broader effects are unclear, but intriguingly, the one CEN-proximal origin that was analyzed (ARS1) replicated later in rif1 $\Delta$ cells, as occurred at all three CENs in $S$. pombe rif1 $\Delta$ cells. It will be interesting to determine whether budding yeast CENs bind Rif1 and require Rif1 for early replication.

Rif1 has now been shown to control replication timing in mammalian cells as well. Yamazaki et al. (2012) showed that depletion of Rif1 altered the replication program in human (HeLa) cells, and Cornacchia et al. (2012) showed that deletion of Rif1 altered replication timing patterns in mouse (MEF [mouse embryonic fibroblast]) cells. Both studies showed loss of the normal cytological pattern of mid-S-phase replication foci with an apparent accumulation of early S-phase patterns, suggesting an increase of early replication events. These 
patterns suggest that Rif1 normally delays the activation of many origins, which fire earlier in Rif1's absence. In addition, analysis of replication timing with BrdU incorporation revealed many early-replicating domains being delayed and late-replicating domains advancing; overall, the replication timing programs were compressed, consistent with a global loss of replication timing control.

\section{Timing mechanism is independent of transcription}

The identification of Fkh1/2 transcription factors as regulators of origin timing recalls early studies of ARS elements that identified trans-acting factor as stimulators of origin function (for review, see DePamphilis 1993). For example, binding sites for ARS-binding factor 1 (Abf1) were identified at a subset of ARSs, including the wellstudied ARS1. Deletion of the Abfl-binding site at chromosomal ARS1 reduced firing efficiency, which was restored by introduction of a binding site for a heterologous protein containing a transcriptional activation domain (Marahrens and Stillman 1992). Subsequent studies indicated that the role of $\mathrm{Abf} 1$ at $A R S 1$ is to maintain a nucleosome-free region for MCM loading, which is required for assembly of competent origins (Lipford and Bell 2001). It has not been reported whether Abf1 affects initiation timing; however, nucleosomal profiles are similar between early and late origins, suggesting that whereas a nucleosome-free region is essential to establish origin competence, it is not a determinant of origin timing (Berbenetz et al. 2010; Eaton et al. 2010).

Origin timing regulation by Fkh1/2 and by Rif1 and Taz1 appears to function by a mechanism distinct from Abf1. Deletion of FKH1 and FKH2 did not affect levels of ORC or MCM loading at origins based on genome-wide ChIP-chip analysis (Knott et al. 2012). Similarly, the timing and level of MCM loading at origins were unaffected by rif1 $1^{+}$or $\operatorname{taz}^{+}$deletion (Hayano et al. 2012; Tazumi et al. 2012). Thus, Fkh1/2, Rif1, and Taz1 do not appear to affect origin licensing.

The involvement of transcription regulators and telomeric chromatin proteins in origin regulation also raised the possibility that transcriptional changes in the mutant cells were responsible for the effects on origin activity. Transcription through an origin sequence can directly disrupt origin activity by interfering with ORC binding and MCM loading (Snyder et al. 1988; Mori and Shirahige 2007; Blitzblau et al. 2012). Thus, changes in transcription might alter origin competence positively or negatively depending on the directionality of transcriptional change. Both fkh1s fkh2s and rif1s yeast and Rif1depleted HeLa cells exhibit substantial changes in transcription (Zhu et al. 2000; Hayano et al. 2012; Knott et al. 2012; Yamazaki et al. 2012). However, no changes in ORC and/or MCM origin-binding levels or -binding time were observed at origins showing deregulated firing in $f k h 1 \Delta$ $f k h 2 \Delta$, rif1s, or taz1s yeast or Rif1-depleted HeLa cells, inconsistent with transcriptional effects on origin competence (Hayano et al. 2012; Knott et al. 2012; Tazumi et al. 2012; Yamazaki et al. 2012). Furthermore, overexpression of C-terminally truncated Fkh2 in fkh1s fkh2 $\Delta$ cells rescued most of the transcriptional deregulation but did not rescue origin deregulation, strongly suggesting that transcriptional changes are not responsible for the effects on origins (Knott et al. 2012). In addition, transcriptome analysis in these cells showed no correlation between any residual changes in transcription and origin deregulation. Transcriptome analysis of $\mathrm{Rif1}^{-/-}$MEFs showed no changes in transcription (Cornacchia et al. 2012). This result appears to contrast with the analysis in yeast and HeLa cells, which, however, were not further scrutinized. Overall, these results suggest that replication timing control, at least by the Fkh1/2, Rif1, and Taz1 mechanisms, is mechanistically independent of transcription.

\section{Regulation of Cdc45-Sld3 loading}

The timing of Cdc45 loading, along with its loading factor, Sld3, corresponds with the differential timing of origin initiation, suggesting that the DDK-dependent recruitment of Cdc45-Sld3 is the temporally regulated step (Aparicio et al. 1999; Kamimura et al. 2001). Furthermore, these studies detected binding of Cdc45 and Sld3 at early origins in G1-arrested budding yeast cells (Fig. 2), leading to the suggestion that Cdc45 might be present in a limiting amount, which is somehow preferentially accessible to early origins (Aparicio et al. 1999). The G1-phase association of Cdc45 with early origins is particularly significant because origin timing is established in G1 phase in budding yeast and mammalian cells (Raghuraman et al. 1997; Dimitrova and Gilbert 1999). The Fkh1/2 study found that Fkh-activated origins were significantly enriched for Cdc45 binding in G1 phase, and, remarkably, this binding was Fkh1/2-dependent (Fig. 1A). Furthermore, Cdc45 binding at CEN-proximal origins was not Fkh1/2-dependent, but instead increased in the absence of Fkh1/2, likely explaining the even earlier activation of CEN-proximal origins in fkh1s fkh2s cells. Thus, Fkh1/2 enhance the ability of Fkh-activated origins to recruit Cdc45 in G1 phase, in anticipation of $S$ phase. Given the dependence of Cdc45 loading on DDK, it is possible that Fkh1/2 act to recruit DDK instead.

Taz1 and Rif1 also regulate the timing of the Cdc45Sld3-loading step (Hayano et al. 2012; Tazumi et al. 2012). In taz1s and rif1s fission yeast cells, Cdc45 and Sld3 binding was advanced at origins that fired earlier in the mutant strains, consistent with inhibitory roles for Taz1 and Rif1 in Cdc45-Sld3 loading (Fig. 1B,C). Similarly, Rif1-depleted HeLa cells showed increased chromatin association of Cdc45 in G1-synchronized cells, suggesting that Rif1 acts prior to replication initiation to inhibit Cdc45 access to origins (Yamazaki et al. 2012). However, Rif1 was required for early S-phase binding of Cdc45 to pericentric origins in fission yeast, which require Rif1 for early firing, suggesting that Rif1 is not simply an inhibitor of Cdc45 loading (Hayano et al. 2012). Further consistent with the idea that Rif1 acts in G1 phase, Rif1 chromatin binding occurs in G1 phase in both fission yeast and mammalian cells (Cornacchia et al. 2012; Hayano et al. 2012; Yamazaki et al. 2012). Interestingly, cytological 


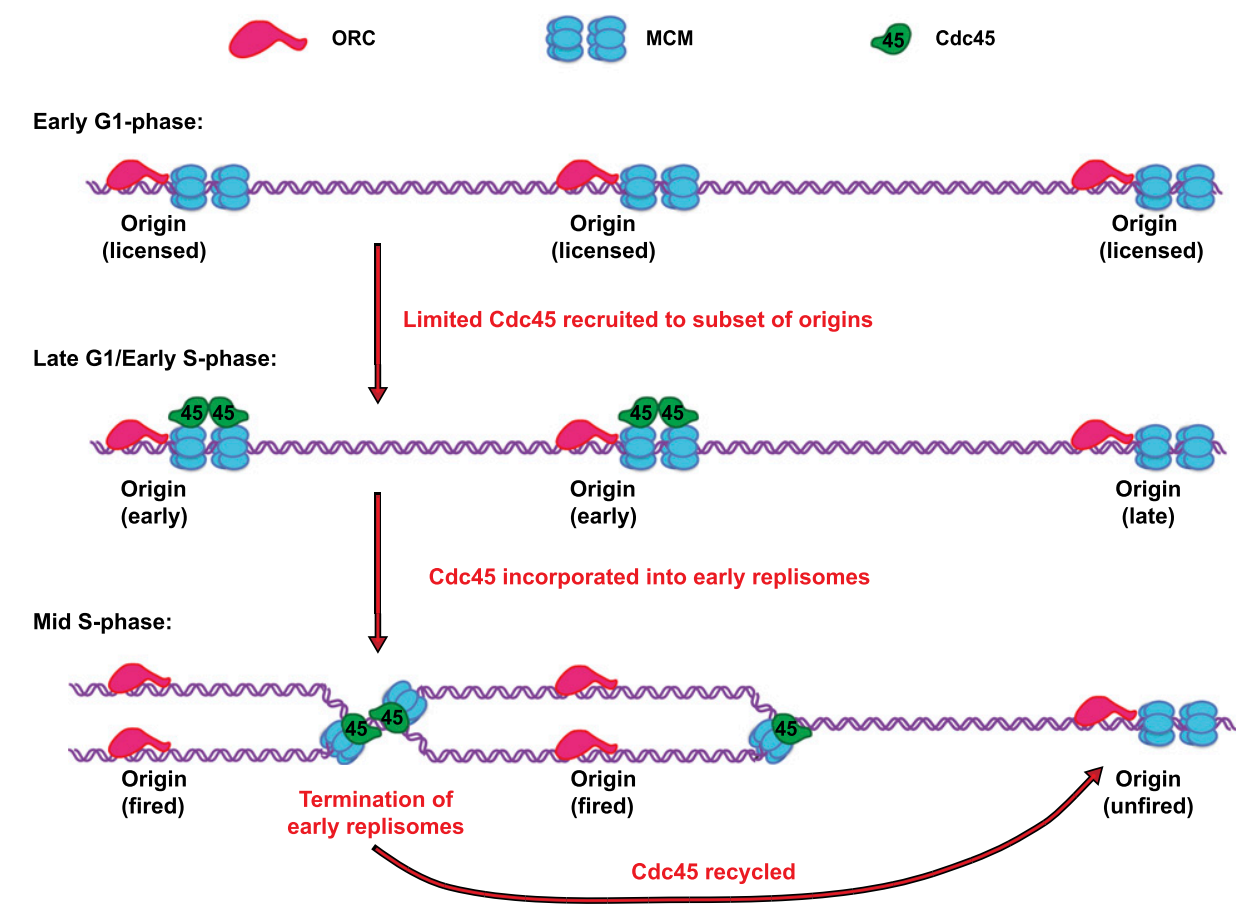

Figure 2. Preferential recruitment and recycling of limiting initiation factors as a timing mechanism. Recruitment of Cdc45 requires Sld3 and DDK activities, which are not shown. Additional initiation factors and replisome components are also excluded for simplicity.

analysis indicated that Rif1 binding preceded replication in MEFs but did not localize with ongoing replication (Cornacchia et al. 2012).

Supporting the idea that Cdc45-Sld3 abundance is limiting and may contribute to replication timing control, studies in several replication systems have provided direct evidence that Cdc45 and a handful of other replication initiation factors are indeed limiting for origin firing in vivo. In Xenopus (frog) egg extracts, only a subset of licensed origins bound Cdc45, and decreasing the abundance of Cdc45 reduced the level of replication (Edwards et al. 2002). In S. pombe, overexpression of Cdc45 or a subunit of DDK (Hsk1 or Dfp1), which is required to load Cdc45-Sld3, increased the level of replication that occurred in early S phase (Patel et al. 2008; Wu and Nurse 2009). In mammalian cells, Cdc 45 was present at low levels relative to licensed origins, and increasing the dosage of Cdc45 resulted in an increased number of replication foci, consistent with an increased number of replisomes (Wong et al. 2011). In S. cerevisiae, overexpression of Cdc45 alone did not advance late origin timing; however, overexpression of Cdc 45 plus its binding partners, Sld3 and Sld7, or overexpression of DDK subunits Cdc7 and Dbf4 did (Tanaka et al. 2011). In a different study in budding yeast, overexpression of Sld3, Dbf4, Sld2, and Dpb11 advanced late origin firing (Mantiero et al. 2011). Furthermore, in the context of overexpressed Sld3, Dbf4, Sld2, and Dpb11, increasing the dosages of Cdc45 and Sld7 increased the overall levels of origin firing, consistent with Cdc45 and Sld7 limiting the total number of active replisomes. Together, these studies indicate that levels of initiation factors, particularly those involved with DDK-dependent Cdc45-Sld3 loading, are rate-limiting for replication initiation.

Limited abundance of Cdc45-Sld3 would allow only a subset of licensed origins to fire upon S-phase entry. Because Cdc45 is a replisome component (Aparicio et al. 1997; Tercero et al. 2000), it would be unavailable to fire additional origins until early replicons terminated and recycled Cdc45, thus creating a mechanism to distribute origin firing temporally (Fig. 2). Replication factor recycling has been implicated as a component of the timing program (Sporbert et al. 2002; Ma et al. 2012). It is also possible that Cdc45-Sld3 loading and recycling are temporally regulated through checkpoint modulation of Sld3 and DDK activities (Lopez-Mosqueda et al. 2010; Zegerman and Diffley 2010), which may establish an upper limit on the number of simultaneously active replisomes. Nevertheless, the timing program is established independently of the checkpoint signaling pathway, at least in yeast (Santocanale and Diffley 1998; Shirahige et al. 1998).

\section{Spatial organization of origins in timing control}

Whereas limiting levels and recycling of initiation factors provide a mechanism to stagger origin firings temporally (Fig. 2), this mechanism does not explain the preferential recruitment of initiation factors to some origins over others, resulting in characteristically distinct firing times at a population level. Indeed, even in the context of overexpressed factors, deletion of $R P D 3$ was required to activate dormant origins, albeit inefficiently, suggesting that Rpd3-dependent chromatin still impedes accessibility 
of replication factors even at increased dosages (Mantiero et al. 2011). Similarly, a subtelomeric region with tethered Dfp1 replicated later than a pericentric region with tethered Dfp1, suggesting that telomeric chromatin hinders replication factors (Hayashi et al. 2009).

The involvement of telomere-binding proteins as global regulators of the replication timing of subtelomeric and many internal origins implies a role for these proteins in the organization or localization of origins within the nucleus. Taz1 and Yku70 are required for localization of telomeres to the nuclear periphery in yeast (Cooper et al. 1997; Laroche et al. 1998). Interestingly, palmitoylation of Rif1 regulates the association of telomeres with the nuclear periphery, suggesting that palmitoylated Rif1 anchors chromatin to the nuclear membrane (Park et al. 2011). Experiments tethering early origins ARS305 and ARS607 to the inner nuclear envelope suggest that positioning to the nuclear periphery is not sufficient to delay origin timing (Zappulla et al. 2002; Ebrahimi et al. 2010). However, both ARS305 and ARS607 are Fkhactivated origins, suggesting that the Fkh1/2 mechanism responsible for the early firing of these origins might be dominant over peripheral tethering, at least in these contexts. In contrast, targeted heterochromatin assembly and peripheral positioning of ARS305 through Sir4 recruitment resulted in delayed firing of ARS305 (Zappulla et al. 2002). Experiments disrupting either SIR chromatin or tethering of telomeres to the nuclear periphery suggest that neither mechanism by itself is essential for late replication (Santocanale et al. 1999; Zappulla et al. 2002; Hiraga et al. 2006). Thus, the combination of peripheral positioning and heterochromatic structure may be required to delay origin firing reliably, particularly in the context of a competing early-firing mechanism.

In mouse and human cells, Rif1 was found in the insoluble nuclear fraction containing nuclear lamins, suggesting association with the nuclear matrix (Cornacchia et al. 2012; Yamazaki et al. 2012). Rif1 depletion in HeLa cells resulted in larger chromatin loops, consistent with a role for Rif1 in anchoring chromatin to nuclear substructures such as the matrix. The $\mathrm{N}$ terminus of Rif 1 from yeast to humans is predicted to contain 14-21 HEAT domain repeats that have been implicated in proteinprotein interactions (Xu et al. 2010), suggesting a potential mechanism to anchor chromatin loops.

The spatial organization of chromosomes and origins has also been implicated in replication timing control by Fkh1/2. Fkh-regulated origins are nonrandomly distributed, with linear groups of Fkh-activated (early) origins frequently separated from linear groups of Fkh-repressed (late) origins (Knott et al. 2012), which is consistent with a previous study that found that origins of similar timings tend to colocalize along budding yeast chromosomes (Raghuraman et al. 2001). These timing domains (up to a few hundred kilobases in length) were lost in fkh1s $f k h 2 \Delta$ cells, suggesting that Fkh1/2 establish replication timing domains between linear groups of origins. Similarly, studies of mammalian genome replication also show that chromosomes are subdivided into replication timing domains (of several hundred kilobases to a few megabases in length) comprising groups of origins with similar timing (Hand 1975; Hiratani et al. 2008). In fact, the replication timing of these domains correlates more strongly with their spatial positioning in the nucleus than with other correlated features such as gene expression or chromatin modifications, suggesting that spatial organization may play a mechanistic role in defining replication timing (Ryba et al. 2010; Yaffe et al. 2010). Loss of Rif1 function in MEFs and HeLa cells led to disruption of replication timing domains, suggesting a role for Rif 1 in defining these spatial domains (Cornacchia et al. 2012; Yamazaki et al. 2012).

Consistent with the mammalian studies of replication domain organization, whole-genome chromosome conformation capture analysis of the yeast genome found enriched interactions between early origins, suggesting that these origins colocalize or associate with each other in the nuclear space (Duan et al. 2010). Further analysis of this data set indicated that Fkh-activated origins formed a distinct interaction cluster from Fkh-repressed origins, suggesting that these groups occupy distinct spaces in the nucleus (Knott et al. 2012). Chromosome conformation capture analysis comparing wild-type and fkh1s fkh2s mutant cells showed that interaction between early origin ARS305 and other Fkh-activated origins (e.g., ARS607) was Fkh1/2-dependent. Together, these results suggest that Fkh1/2 regulate the spatial organization of origins, which controls replication timing. A role for Fkh1/2 in mediating long-range interaction between chromosomal loci is supported by the role of Fkh1 in regulating donor preference during mating type switching in budding yeast, which involves recombination between distal chromosomal loci (Sun et al. 2002).

Fkh1/2 were shown to interact physically with ORC by coimmunoprecipitation (Knott et al. 2012). Hence, Fkh1/2 bound at one origin might interact in trans with ORC at another origin, with multiple such interactions forming a cluster of origins. This mechanism does not necessarily exclude origins lacking Fkh1/2-binding sites. As a group, Fkh-activated origins are enriched for Fkh1/2 consensus binding sites; however, existing ChIP-chip analyses did not detect Fkh1/2 binding at many consensus sites, so it is not clear whether Fkh1/2 uniformly bind Fkh-activated origins (Harbison et al. 2004; MacIsaac et al. 2006). Renewed analysis of Fkh1/2 binding, particularly during G1 phase, should provide important insight.

A compelling alternative mechanism for Fkh1/2 to mediate interactions in trans specifically between Fkhl/ 2-bound origins involves dimerization of Fkh1/2. Crystal structures of the human FoxP2 and FoxP3 forkhead DNAbinding domains revealed their ability to form domainswapped dimers, suggesting that these proteins might bridge two DNA molecules (Stroud et al. 2006; Bandukwala et al. 2011). Furthermore, mutations that disrupt potential dimerization of FoxP3 have phenotypic consequences, supporting a functional role for dimerization. Structural modeling of yeast Fkh1/2 based on the FoxP2 and FoxP3 structures predicts that Fkh1/2 also dimerize, and mutations of Fkh1/2 predicted to disrupt dimerization deregulate origin timing, supporting a functional 
requirement for dimerization (A Ostrow, R Kalhor, L Chen, and $\mathrm{O}$ Aparicio, unpubl.). Dimerization of Fkh1/2 might enable clustering specifically between Fkh1/2-bound origins, and the presence of multiple Fkh1/2-binding sites at an origin, as demonstrated at ARS305 (Knott et al. 2012), would further permit the clustering of multiple origins together (Fig. 3).

The elucidation of mechanisms promoting early firing helps explain the robustness of the replication timing program, at least in budding yeast. These findings support a model based on competition between origins for limiting replication factors where $\mathrm{Fkhl} / 2$ promote early firing by binding specific origins and drawing these origins into clusters or preferred locations in G1 phase (Fig. 3). Clustering of licensed origins having an affinity for Cdc45 and other factors may create a cooperative effect that locally aggregates these factors in anticipation of initiation. Alternatively, Fkh1/2 might position origins with replication factors localized by an unknown mechanism. CENs (at least in budding yeast) might act similarly to promote early firing of proximal origins due to the characteristic clustering and subnuclear positioning of CENs (Jin et al. 1998). Present support for the idea that limiting factors are localized within the nucleus is indirect, based on Cdc45's association with origins that are themselves distinctly localized according to the chromosome conformation capture studies; nevertheless, this feature is not essential for the model. Meanwhile, Yku70, Taz1, and Rif1 generally promote later firing through origin localization to the nuclear periphery and/or through heterochromatic chromatin assembly, effectively sequestering these origins from initiation factors (Fig. 3). The presence of opposing mechanisms should magnify the effect of competition between individual origins for limiting replication initiation factors. In addition, the limited availability of multiple initiation factors would likely enhance the differences in origins' probabilities of recruiting all of the necessary factors for initiation and provide a strong kinetic advantage to some origins over others.

Initiation transforms early origin clusters into the replication foci that are first observed during $S$ phase. As replication ensues, significant mobilization of chromosomes occurs as DNA is spooled through replisomes within the replication foci (Ma et al. 1998; Leonhardt et al. 2000). This may relocate other, unfired origins to the proximity of the replication foci, where they may capture limiting initiation factors as these are released from terminating, early replicons (Sporbert et al. 2002; Ma et al. 2012). Eventually, telomeres and other sequences associated with the nuclear periphery are released to gain access to replication factors (Heun et al. 2001; Ebrahimi and Donaldson 2008). In mammalian cells, however, replication foci assemble near the nuclear periphery later in $S$ phase, suggesting a relocation of replication factors rather than the chromosomal loci themselves (for review, see Berezney et al. 2000).

Interestingly, subtelomeric origins became even later firing in fkh1s fkh2s yeast cells (Knott et al. 2012), suggesting that their relocation from the nuclear periphery depends on prior replication events, such as the firing of internal earlier origins, perhaps proximal to the sub-

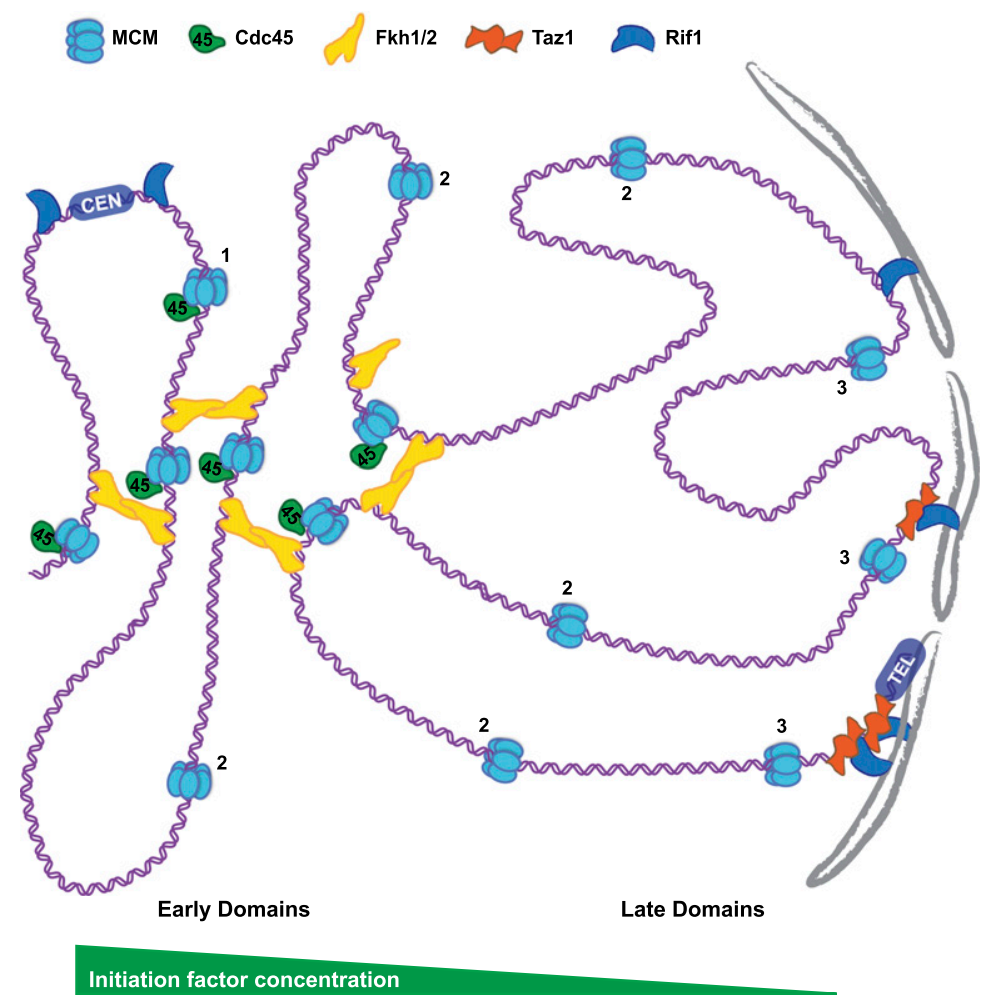

Figure 3. Replication timing model based on spatial organization of origins and limiting factor accumulation. Licensed origins are represented by a single MCM hexamer for simplicity. In G1 phase, interactions between Fkh1/2-bound origins create clusters that localize with Cdc45 and likely other limiting factors (not shown), creating a high probability of Cdc 45 recruitment (although not all clustered origins are expected to recruit Cdc45 early in every cell). A Cdc45 gradient may form due to cooperative recruitment by clustered origins or another mechanism but remains speculative. CEN localizes proximal origin (1) with Cdc45, perhaps with Rif1 involvement, and/or clustering with other CENs (not shown). At other loci, Rif1 anchors Taz1and Rif1-bound sites to the nuclear membrane, thereby localizing proximal origins to the nuclear periphery, sequestered from Cdc45 accumulation. Local heterochromatin may also contribute to Cdc45 exclusion from these domains. The cluster of Cdc45-bound origins, likely including origins from other chromosomes (not shown), establishes a replication focus in early S phase comprising these multiple replisomes. Replication draws flanking origins (2) toward the focus, where they access Cdc45, possibly recycled from earlier replisomes, and fire or replicate passively. Eventually, peripheral origins (3) relocate and fire or replicate passively, although in mammalian cells, the replication factors instead relocate to the periphery. 
telomeric regions. This repositioning may increase the probability that these origins will fire during this later period. In addition, the diminishing numbers of unfired origins competing for limiting factors over time should also increase the chance that these origins will eventually fire (Lucas et al. 2000; Goldar et al. 2008; de Moura et al. 2010; Rhind et al. 2010). The probability of passive replication also increases over time until firing or passive replication of all origins has occurred. This model can explain how some late origins fire efficiently in late $S$ phase despite firing inefficiently in early $S$ phase.

In the context of the above model, deletion of FKH1 and $F K H 2, \operatorname{taz} 1^{+}$, or rif $1^{+}$would alter the favorable or unfavorable positioning of origins bound directly by these proteins and delay or advance their firing time, respectively. However, deletion of these factors also impacted many origins where binding of the corresponding protein was not detected. This might reflect the repositioning of these loci due to their chromosomal cis-linkage with directly bound sites. This "cis-effect" might be expected to increase the likelihood that certain origins will access the pool of limiting factors and decrease this likelihood at other origins. In addition, reduced firing of early origins (e.g., Fkh-activated origins in fkh1s fkh2s cells) may contribute to earlier firing of other origins (e.g., CENproximal and Fkh-repressed origins in fkh1s fkh2s cells) by reducing competition for limiting factors. Conversely, the earlier activation of LE origins in rif1A cells may increase competition for limiting factors and account for the delayed activation of EL origins.

This generic model combines the early-determining CEN and Fkh1/2 mechanisms shown in budding yeast with the late-determining Yku70, Taz1, and Rif1 mechanisms, of which at least the Rif1 mechanism seems to be broadly conserved. It remains to be determined whether the Fkh1/2 mechanism is conserved in $S$. pombe and other eukaryotes. Despite conservation of Fkh2 in $S$. pombe, its sequence suggests an inability to dimerize, which appears to be required for origin regulation (O Aparicio, unpubl.). The absence of this mechanism could explain why origins in $S$. pombe generally fire more stochastically and less efficiently than in S. cerevisiae (Dai et al. 2005; Heichinger et al. 2006; Patel et al. 2006). Indeed, most internal origin firing appears to be more stochastic in S. cerevisiae cells lacking FKH1 and FKH2 (Knott et al. 2012). On the other hand, the role of Fox proteins as pioneer transcription factors during early mammalian development suggests a potential link between the changes in transcription and replication timing that occur during early differentiation (for review, see Gilbert et al. 2010; Lalmansingh et al. 2012). The ability of FoxP2 and FoxP3 to dimerize and potentially mediate long-range chromosomal interactions supports the possibility that these proteins regulate replication timing in human cells.

\section{Future prospects and implications}

These recent studies begin to reveal a more defined picture of the replication process, particularly in the identity of those factors that determine when, and perhaps where, distinct chromosomal domains replicate. The identification of new factors in yeasts provides important candidates to study the mechanisms and significance of replication timing in metazoan cells. Moreover, Fkh1/2 and Rif1 are excellent candidates for organizers of replication foci where clusters of replication origins may act coordinately, but which remain imprecisely defined. Indeed, the foci are dynamic structures that likely must be disassembled to permit replication to progress without undue spatial constraints. Also exciting is the potential identification of proteins that regulate the three-dimensional organization of chromosomes, which is emerging as an important mechanism of genome regulation, having been implicated in regulation of transcription, recombination, genome stability, and epigenetic inheritance (for review, see Misteli 2007). Once again, studies in yeasts are elucidating a fundamental mechanism of genome regulation in all eukaryotes. This is worth celebrating with your favorite yeast product.

\section{Acknowledgments}

Work in the Aparicio laboratory has been supported by NIH grant R01-GM065494. Apologies to those whose work was not included due to limitations of space or scope.

\section{References}

Aladjem MI. 2007. Replication in context: Dynamic regulation of DNA replication patterns in metazoans. Nat Rev Genet 8: 588-600.

Aparicio OM, Weinstein DM, Bell SP. 1997. Components and dynamics of DNA replication complexes in $S$. cerevisiae: Redistribution of MCM proteins and Cdc45p during S phase. Cell 91: 59-69.

Aparicio OM, Stout AM, Bell SP. 1999. Differential assembly of Cdc45p and DNA polymerases at early and late origins of DNA replication. Proc Natl Acad Sci 96: 9130-9135.

Aparicio JG, Viggiani CJ, Gibson DG, Aparicio OM. 2004. The Rpd3-Sin3 histone deacetylase regulates replication timing and enables intra-S origin control in Saccharomyces cerevisiae. Mol Cell Biol 24: 4769-4780.

Bandukwala HS, Wu Y, Feuerer M, Chen Y, Barboza B, Ghosh S, Stroud JC, Benoist C, Mathis D, Rao A, et al. 2011. Structure of a domain-swapped FOXP3 dimer on DNA and its function in regulatory T cells. Immunity 34: 479-491.

Bannister AJ, Zegerman P, Partridge JF, Miska EA, Thomas JO, Allshire RC, Kouzarides T. 2001. Selective recognition of methylated lysine 9 on histone $\mathrm{H} 3$ by the HP1 chromo domain. Nature 410: 120-124.

Bell SP, Dutta A. 2002. DNA replication in eukaryotic cells. Annu Rev Biochem 71: 333-374.

Berbenetz NM, Nislow C, Brown GW. 2010. Diversity of eukaryotic DNA replication origins revealed by genomewide analysis of chromatin structure. PLoS Genet 6: e1001092.

Berezney R, Dubey DD, Huberman JA. 2000. Heterogeneity of eukaryotic replicons, replicon clusters, and replication foci. Chromosoma 108: 471-484.

Blitzblau HG, Chan CS, Hochwagen A, Bell SP. 2012. Separation of DNA replication from the assembly of break-competent meiotic chromosomes. PLoS Genet 8: e1002643.

Burgess DJ. 2011. Genomic instability: Close-up on cancer copy number alterations. Nat Rev Genet 13: 5 . 
Cooper JP, Nimmo ER, Allshire RC, Cech TR. 1997. Regulation of telomere length and function by a Myb-domain protein in fission yeast. Nature 385: 744-747.

Cornacchia D, Dileep V, Quivy JP, Foti R, Tili F, SantarellaMellwig R, Antony C, Almouzni G, Gilbert DM, Buonomo SB. 2012. Mouse Rif1 is a key regulator of the replicationtiming programme in mammalian cells. EMBO J 31: 36783690.

Cosgrove AJ, Nieduszynski CA, Donaldson AD. 2002. Ku complex controls the replication time of DNA in telomere regions. Genes Dev 16: 2485-2490.

Cvetic C, Walter JC. 2005. Eukaryotic origins of DNA replication: Could you please be more specific? Semin Cell Dev Biol 16: $343-353$.

Czajkowsky DM, Liu J, Hamlin JL, Shao Z. 2008. DNA combing reveals intrinsic temporal disorder in the replication of yeast chromosome VI. J Mol Biol 375: 12-19.

Dai J, Chuang RY, Kelly TJ. 2005. DNA replication origins in the Schizosaccharomyces pombe genome. Proc Natl Acad Sci 102: 337-342.

de Moura AP, Retkute R, Hawkins M, Nieduszynski CA. 2010. Mathematical modelling of whole chromosome replication. Nucleic Acids Res 38: 5623-5633.

DePamphilis ML. 1993. Eukaryotic DNA replication: Anatomy of an origin. Annu Rev Biochem 62: 29-63.

Dimitrova DS, Gilbert DM. 1999. The spatial position and replication timing of chromosomal domains are both established in early G1 phase. Mol Cell 4: 983-993.

Duan Z, Andronescu M, Schutz K, McIlwain S, Kim YJ, Lee C, Shendure I, Fields S, Blau CA, Noble WS. 2010. A threedimensional model of the yeast genome. Nature 465: 363 367.

Dubey DD, Davis LR, Greenfeder SA, Ong LY, Zhu JG, Broach JR, Newlon CS, Huberman JA. 1991. Evidence suggesting that the ARS elements associated with silencers of the yeast mating-type locus HML do not function as chromosomal DNA replication origins. Mol Cell Biol 11: 5346-5355.

Eaton ML, Galani K, Kang S, Bell SP, MacAlpine DM. 2010. Conserved nucleosome positioning defines replication origins. Genes Dev 24: 748-753.

Ebrahimi H, Donaldson AD. 2008. Release of yeast telomeres from the nuclear periphery is triggered by replication and maintained by suppression of Ku-mediated anchoring. Genes Dev 22: 3363-3374.

Ebrahimi H, Robertson ED, Taddei A, Gasser SM, Donaldson $A D$, Hiraga S. 2010. Early initiation of a replication origin tethered at the nuclear periphery. J Cell Sci 123: 1015-1019.

Edwards MC, Tutter AV, Cvetic C, Gilbert CH, Prokhorova TA, Walter JC. 2002. MCM2-7 complexes bind chromatin in a distributed pattern surrounding the origin recognition complex in Xenopus egg extracts. I Biol Chem 277: 3304933057.

Fangman WL, Brewer BJ. 1991. Activation of replication origins within yeast chromosomes. Annu Rev Cell Biol 7: 375-402.

Ferguson BM, Fangman WL. 1992. A position effect on the time of replication origin activation in yeast. Cell 68: 333-339.

Friedman KL, Brewer BJ. 1995. Analysis of replication intermediates by two-dimensional agarose gel electrophoresis. Methods Enzymol 262: 613-627.

Friedman KL, Diller JD, Ferguson BM, Nyland SV, Brewer BJ, Fangman WL. 1996. Multiple determinants controlling activation of yeast replication origins late in S phase. Genes Dev 10: 1595-1607.

Friedman KL, Brewer BJ, Fangman WL. 1997. Replication profile of Saccharomyces cerevisiae chromosome VI. Genes Cells 2: 667-678.
Gilbert DM, Takebayashi SI, Ryba T, Lu J, Pope BD, Wilson KA, Hiratani I. 2010. Space and time in the nucleus: Developmental control of replication timing and chromosome architecture. Cold Spring Harb Symp Quant Biol 75: 143-153.

Goldar A, Labit H, Marheineke K, Hyrien O. 2008. A dynamic stochastic model for DNA replication initiation in early embryos. PLOS ONE 3: e2919.

Gondor A, Ohlsson R. 2009. Replication timing and epigenetic reprogramming of gene expression: A two-way relationship? Nat Rev Genet 10: 269-276.

Hand R. 1975. Regulation of DNA replication on subchromosomal units of mammalian cells. J Cell Biol 64: 89-97.

Harbison CT, Gordon DB, Lee TI, Rinaldi NJ, Macisaac KD, Danford TW, Hannett NM, Tagne JB, Reynolds DB, Yoo J, et al. 2004. Transcriptional regulatory code of a eukaryotic genome. Nature 431: 99-104.

Hayano M, Kanoh Y, Matsumoto S, Renard-Guillet C, Shirahige K, Masai H. 2012. Rif1 is a global regulator of timing of replication origin firing in fission yeast. Genes Dev 26: 137150.

Hayashi MT, Takahashi TS, Nakagawa T, Nakayama J, Masukata H. 2009. The heterochromatin protein Swi6/HP1 activates replication origins at the pericentromeric region and silent mating-type locus. Nat Cell Biol 11: 357-362.

Heichinger C, Penkett CI, Bahler J, Nurse P. 2006. Genome-wide characterization of fission yeast DNA replication origins. EMBO I 25: 5171-5179.

Heller RC, Kang S, Lam WM, Chen S, Chan CS, Bell SP. 2011. Eukaryotic origin-dependent DNA replication in vitro reveals sequential action of DDK and S-CDK kinases. Cell 146: 80-91.

Herrick J. 2011. Genetic variation and DNA replication timing, or why is there late replicating DNA? Evolution 65: 30313047.

Heun P, Laroche T, Raghuraman MK, Gasser SM. 2001. The positioning and dynamics of origins of replication in the budding yeast nucleus. J Cell Biol 152: 385-400.

Hiraga S, Robertson ED, Donaldson AD. 2006. The Ctf18 RFClike complex positions yeast telomeres but does not specify their replication time. EMBO J 25: 1505-1514.

Hiratani I, Ryba T, Itoh M, Yokochi T, Schwaiger M, Chang CW, Lyou Y, Townes TM, Schubeler D, Gilbert DM. 2008. Global reorganization of replication domains during embryonic stem cell differentiation. PLOS Biol 6: e245.

Huberman JA. 1997. Mapping replication origins, pause sites, and termini by neutral/alkaline two-dimensional gel electrophoresis. Methods 13: 247-257.

Jin Q, Trelles-Sticken E, Scherthan H, Loidl J. 1998. Yeast nuclei display prominent centromere clustering that is reduced in nondividing cells and in meiotic prophase. J Cell Biol 141: 21-29.

Kalejta RF, Hamlin JL. 1996. Composite patterns in neutral/ neutral two-dimensional gels demonstrate inefficient replication origin usage. Mol Cell Biol 16: 4915-4922.

Kamimura Y, Tak YS, Sugino A, Araki H. 2001. Sld3, which interacts with Cdc45 (Sld4), functions for chromosomal DNA replication in Saccharomyces cerevisiae. EMBO I 20: 2097-2107.

Kanoh J, Ishikawa F. 2001. spRap1 and spRif1, recruited to telomeres by Taz1, are essential for telomere function in fission yeast. Curr Biol 11: 1624-1630.

Knott SR, Viggiani CJ, Tavaré S, Aparicio OM. 2009. Genomewide replication profiles indicate an expansive role for Rpd3L in regulating replication initiation timing or efficiency, and reveal genomic loci of Rpd3 function in Saccharomyces cerevisiae. Genes Dev 23: 1077-1090. 
Knott SR, Peace JM, Ostrow AZ, Gan Y, Rex AE, Viggiani CJ, Tavare S, Aparicio OM. 2012. Forkhead transcription factors establish origin timing and long-range clustering in $S$. cerevisiae. Cell 148: 99-111.

Koren A, Tsai HJ, Tirosh I, Burrack LS, Barkai N, Berman J. 2010. Epigenetically-inherited centromere and neocentromere DNA replicates earliest in S-phase. PLoS Genet 6: e1001068.

Labib K. 2010. How do Cdc7 and cyclin-dependent kinases trigger the initiation of chromosome replication in eukaryotic cells? Genes Dev 24: 1208-1219.

Lalmansingh AS, Karmakar S, Jin Y, Nagaich AK. 2012. Multiple modes of chromatin remodeling by Forkhead box proteins. Biochim Biophys Acta 1819: 707-715.

Laroche T, Martin SG, Gotta M, Gorham HC, Pryde FE, Louis EJ, Gasser SM. 1998. Mutation of yeast Ku genes disrupts the subnuclear organization of telomeres. Curr Biol 8: 653-656.

Leonhardt H, Rahn HP, Weinzierl P, Sporbert A, Cremer T, Zink D, Cardoso MC. 2000. Dynamics of DNA replication factories in living cells. J Cell Biol 149: 271-280.

Lian HY, Robertson ED, Hiraga S, Alvino GM, Collingwood D, McCune HJ, Sridhar A, Brewer BJ, Raghuraman MK, Donaldson AD. 2011. The effect of $\mathrm{Ku}$ on telomere replication time is mediated by telomere length but is independent of histone tail acetylation. Mol Biol Cell 22: 1753-1765.

Lipford JR, Bell SP. 2001. Nucleosomes positioned by ORC facilitate the initiation of DNA replication. Mol Cell 7: 21-30.

Lopez-Mosqueda J, Maas NL, Jonsson ZO, Defazio-Eli LG, Wohlschlegel J, Toczyski DP. 2010. Damage-induced phosphorylation of Sld3 is important to block late origin firing. Nature 467: 479-483.

Lucas I, Chevrier-Miller M, Sogo JM, Hyrien O. 2000. Mechanisms ensuring rapid and complete DNA replication despite random initiation in Xenopus early embryos. J Mol Biol 296: 769-786.

Ma H, Samarabandu J, Devdhar RS, Acharya R, Cheng PC, Meng C, Berezney R. 1998. Spatial and temporal dynamics of DNA replication sites in mammalian cells. J Cell Biol 143: 14151425.

Ma E, Hyrien O, Goldar A. 2012. Do replication forks control late origin firing in Saccharomyces cerevisiae? Nucleic Acids Res 40: 2010-2019.

MacIsaac KD, Wang T, Gordon DB, Gifford DK, Stormo GD, Fraenkel E. 2006. An improved map of conserved regulatory sites for Saccharomyces cerevisiae. BMC Bioinformatics 7: 113.

Mantiero D, Mackenzie A, Donaldson A, Zegerman P. 2011. Limiting replication initiation factors execute the temporal programme of origin firing in budding yeast. $E M B O I$ 30: 4805-4814.

Marahrens Y, Stillman B. 1992. A yeast chromosomal origin of replication defined by multiple functional elements. Science 255: 817-823.

Marcand S, Wotton D, Gilson E, Shore D. 1997. Raplp and telomere length regulation in yeast. Ciba Found Symp 211: 76-93.

Mendez J. 2009. Temporal regulation of DNA replication in mammalian cells. Crit Rev Biochem Mol Biol 44: 343-351.

Misteli T. 2007. Beyond the sequence: Cellular organization of genome function. Cell 128: 787-800.

Mori S, Shirahige K. 2007. Perturbation of the activity of replication origin by meiosis-specific transcription. I Biol Chem 282: 4447-4452.

Nakayama J, Rice JC, Strahl BD, Allis CD, Grewal SI. 2001. Role of histone H3 lysine 9 methylation in epigenetic control of heterochromatin assembly. Science 292: 110-113.
Natsume T, Tanaka TU. 2010. Spatial regulation and organization of DNA replication within the nucleus. Chromosome Res 18: 7-17.

Newlon CS, Theis JF. 1993. The structure and function of yeast ARS elements. Curr Opin Genet Dev 3: 752-758.

Newlon CS, Collins I, Dershowitz A, Deshpande AM, Greenfeder SA, Ong LY, Theis JF. 1993. Analysis of replication origin function in chromosome III of Saccharomyces cerevisiae. Cold Spring Harb Symp Quant Biol 58: 415-423.

Park S, Patterson EE, Cobb J, Audhya A, Gartenberg MR, Fox CA. 2011. Palmitoylation controls the dynamics of buddingyeast heterochromatin via the telomere-binding protein Rif1. Proc Natl Acad Sci 108: 14572-14577.

Patel PK, Arcangioli B, Baker SP, Bensimon A, Rhind N. 2006. DNA replication origins fire stochastically in fission yeast. Mol Biol Cell 17: 308-316.

Patel PK, Kommajosyula N, Rosebrock A, Bensimon A, Leatherwood J, Bechhoefer J, Rhind N. 2008. The Hsk1(Cdc7) replication kinase regulates origin efficiency. Mol Biol Cell 19: $5550-5558$

Pohl TJ, Brewer BJ, Raghuraman MK. 2012. Functional centromeres determine the activation time of pericentric origins of DNA replication in Saccharomyces cerevisiae. PLoS Genet 8: e1002677.

Poloumienko A, Dershowitz A, De J, Newlon CS. 2001. Completion of the replication map of Saccharomyces cerevisiae chromosome III. Mol Biol Cell 12: 3317-3327.

Raghuraman MK, Brewer BJ, Fangman WL. 1997. Cell cycledependent establishment of a late replication program. Science 276: 806-809.

Raghuraman MK, Winzeler EA, Collingwood D, Hunt S, Wodicka L, Conway A, Lockhart DJ, Davis RW, Brewer BJ, Fangman WL. 2001. Replication dynamics of the yeast genome. Science 294: 115-121.

Rhind N. 2006. DNA replication timing: Random thoughts about origin firing. Nat Cell Biol 8: 1313-1316.

Rhind N, Yang SC, Bechhoefer J. 2010. Reconciling stochastic origin firing with defined replication timing. Chromosome Res 18: 35-43.

Ryba T, Hiratani I, Lu J, Itoh M, Kulik M, Zhang J, Schulz TC, Robins AJ, Dalton S, Gilbert DM. 2010. Evolutionarily conserved replication timing profiles predict long-range chromatin interactions and distinguish closely related cell types. Genome Res 20: 761-770.

Santocanale C, Diffley JF. 1998. A Mec1- and Rad53-dependent checkpoint controls late-firing origins of DNA replication. Nature 395: 615-618.

Santocanale C, Sharma K, Diffley JF. 1999. Activation of dormant origins of DNA replication in budding yeast. Genes Dev 13: 2360-2364.

Shirahige K, Hori Y, Shiraishi K, Yamashita M, Takahashi K, Obuse C, Tsurimoto T, Yoshikawa H. 1998. Regulation of DNA-replication origins during cell-cycle progression. $\mathrm{Na}$ ture 395: 618-621.

Shore D, Bianchi A. 2009. Telomere length regulation: Coupling DNA end processing to feedback regulation of telomerase. EMBO J 28: 2309-2322.

Snyder M, Sapolsky RJ, Davis RW. 1988. Transcription interferes with elements important for chromosome maintenance in Saccharomyces cerevisiae. Mol Cell Biol 8: 2184 2194.

Sporbert A, Gahl A, Ankerhold R, Leonhardt H, Cardoso MC. 2002. DNA polymerase clamp shows little turnover at established replication sites but sequential de novo assembly at adjacent origin clusters. Mol Cell 10: 13551365. 
Stevenson JB, Gottschling DE. 1999. Telomeric chromatin modulates replication timing near chromosome ends. Genes Dev 13: 146-151.

Stinchcomb DT, Struhl K, Davis RW. 1979. Isolation and characterisation of a yeast chromosomal replicator. Nature 282: 39-43.

Stroud JC, Wu Y, Bates DL, Han A, Nowick K, Paabo S, Tong H, Chen L. 2006. Structure of the forkhead domain of FOXP2 bound to DNA. Structure 14: 159-166.

Sun K, Coic E, Zhou Z, Durrens P, Haber JE. 2002. Saccharomyces forkhead protein Fkh1 regulates donor preference during mating-type switching through the recombination enhancer. Genes Dev 16: 2085-2096.

Tanaka S, Nakato R, Katou Y, Shirahige K, Araki H. 2011. Origin association of Sld3, Sld7, and Cdc45 proteins is a key step for determination of origin-firing timing. Curr Biol 21: 2055-2063.

Tazumi A, Fukuura M, Nakato R, Kishimoto A, Takenaka T, Ogawa S, Song JH, Takahashi TS, Nakagawa T, Shirahige K, et al. 2012. Telomere-binding protein Tazl controls global replication timing through its localization near late replication origins in fission yeast. Genes Dev 26: 2050-2062.

Tercero JA, Labib K, Diffley JF. 2000. DNA synthesis at individual replication forks requires the essential initiation factor Cdc45p. EMBO I 19: 2082-2093.

van Brabant AJ, Hunt SY, Fangman WL, Brewer BJ. 1998 Identifying sites of replication initiation in yeast chromosomes: Looking for origins in all the right places. Electrophoresis 19: 1239-1246.

Vogelauer M, Rubbi L, Lucas I, Brewer BJ, Grunstein M. 2002. Histone acetylation regulates the time of replication origin firing. Mol Cell 10: 1223-1233.

Vujcic M, Miller CA, Kowalski D. 1999. Activation of silent replication origins at autonomously replicating sequence elements near the HML locus in budding yeast. Mol Cell Biol 19: 6098-6109.

Wong PG, Winter SL, Zaika E, Cao TV, Oguz U, Koomen JM, Hamlin JL, Alexandrow MG. 2011. Cdc45 limits replicon usage from a low density of preRCs in mammalian cells. PLOS ONE 6: e17533.

Wu PY, Nurse P. 2009. Establishing the program of origin firing during S phase in fission yeast. Cell 136: 852-864.

Xu D, Muniandy P, Leo E, Yin J, Thangavel S, Shen X, Ii M, Agama K, Guo R, Fox D III, et al. 2010. Rif1 provides a new DNA-binding interface for the Bloom syndrome complex to maintain normal replication. EMBO J 29: 3140-3155.

Yabuki N, Terashima H, Kitada K. 2002. Mapping of early firing origins on a replication profile of budding yeast. Genes Cells 7: 781-789.

Yaffe E, Farkash-Amar S, Polten A, Yakhini Z, Tanay A, Simon I. 2010. Comparative analysis of DNA replication timing reveals conserved large-scale chromosomal architecture. PLOS Genet 6: e1001011.

Yamashita M, Hori Y, Shinomiya T, Obuse C, Tsurimoto T, Yoshikawa H, Shirahige K. 1997. The efficiency and timing of initiation of replication of multiple replicons of Saccharomyces cerevisiae chromosome VI. Genes Cells 2: 655-665.

Yamazaki S, Ishii A, Kanoh Y, Oda M, Nishito Y, Masai H. 2012. Rif1 regulates the replication timing domains on the human genome. EMBO J 31: 3667-3677.

Yang SC, Rhind N, Bechhoefer J. 2010. Modeling genome-wide replication kinetics reveals a mechanism for regulation of replication timing. Mol Syst Biol 6: 404.

Yompakdee C, Huberman JA. 2004. Enforcement of late replication origin firing by clusters of short G-rich DNA sequences. J Biol Chem 279: 42337-42344.
Zappulla DC, Sternglanz R, Leatherwood J. 2002. Control of replication timing by a transcriptional silencer. Curr Biol 12: 869-875.

Zegerman P, Diffley JF. 2010. Checkpoint-dependent inhibition of DNA replication initiation by Sld3 and Dbf4 phosphorylation. Nature 467: 474-478.

Zhu G, Spellman PT, Volpe T, Brown PO, Botstein D, Davis TN, Futcher B. 2000. Two yeast forkhead genes regulate the cell cycle and pseudohyphal growth. Nature 406: 90-94. 


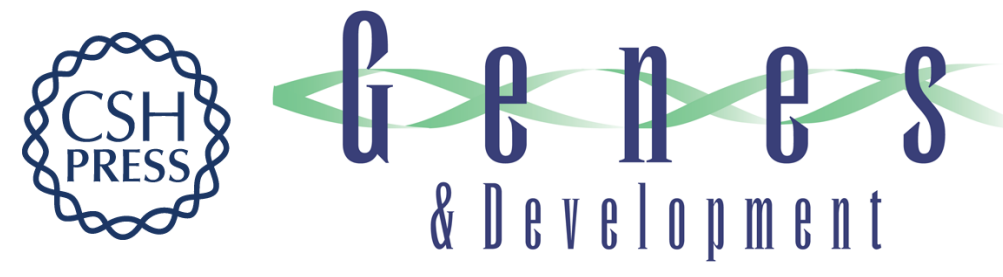

\section{Location, location, location: it's all in the timing for replication origins}

Oscar M. Aparicio

Genes Dev. 2013, 27:

Access the most recent version at doi:10.1101/gad.209999.112

References This article cites 111 articles, 41 of which can be accessed free at: http://genesdev.cshlp.org/content/27/2/117.full.html\#ref-list-1

License

Email Alerting Receive free email alerts when new articles cite this article - sign up in the box at the top Service right corner of the article or click here.

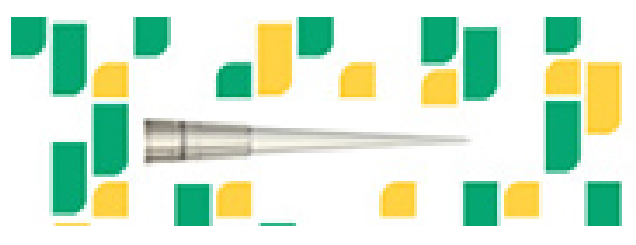

Focused on your science. 